\title{
Correlation of elastic characteristics of breast lesions with the expression of Smad2/3
}

\author{
Meng-Ke Zhang ${ }^{1 \#}$, Shi-Yu Li ${ }^{1 \#}$, Shao-Wei Xue ${ }^{1 \#}$, Gang Liu ${ }^{2}$, Zhi-Li Wang ${ }^{1}$ \\ ${ }^{1}$ Department of Ultrasound, The First Medical Center of PLA General Hospital, Beijing, China; ${ }^{2}$ Department of Radiology, The First Medical \\ Center of PLA General Hospital, Beijing, China \\ Contributions: (I) Conception and design: G Liu, ZL Wang; (II) Administrative support: G Liu, ZL Wang; (III) Provision of study materials or \\ patients: MK Zhang, SY Li, SW Xue; (IV) Collection and assembly of data: MK Zhang, SY Li; (V) Data analysis and interpretation: MK Zhang, SY \\ Li; (VI) Manuscript writing: All authors; (VII) Final approval of manuscript: All authors. \\ \#These authors contributed equally to this work. \\ Correspondence to: Gang Liu. Department of Radiology, The First Medical Center of PLA General Hospital, 28 Fuxing Road, Beijing, 100853, China. \\ Email: 13611245784@12.com; Zhi-Li Wang. Department of Ultrasound, The First Medical Center of PLA General Hospital, 28 Fuxing Road, \\ Beijing 100853, China. Email: wzllg@sina.com.
}

Background: To analyze the relationship between elastic characteristics and the expression of Smad2/3 in breast lesions.

Methods: Between April 2018 and October 2018, 135 lesions from 130 patients who underwent shear wave elastography before surgical excision or biopsy were included in the study. The shear wave elasticity features of the lesions, expression of Smad2/3 of the specimens, and their combined diagnostic efficacy was analyzed. Results: Of the 135 lesions, 51 were malignant and 84 were benign. The elasticity ratio of lesions to peripheral parenchyma, maximum elasticity, mean elasticity, prevalent rate of "stiff rim sign", and the expression level of Smad2/3 in the malignant pathological changes were obviously superior to those with benign pathological change $(\mathrm{P}<0.001)$. The $\mathrm{Smad} 2 / 3$ expression level had a positive correlation with the maximum, average elasticity, and the elastic ratio of lesions to peripheral parenchyma $(r=0.657,0.640$, and 0.470 , respectively, $\mathrm{P}<0.001)$. The expression of $\mathrm{Smad} 2 / 3$ in lesions with "stiff rim sign" was statistically higher than that in lesions without "stiff rim sign" $(\mathrm{P}<0.001)$. Moreover, the combination of Smad2/3 expression and "stiff rim sign" was shown to greatly raise the sensitivity (100\%) and accuracy (94.56\%) in the differential diagnosis of mammary gland disease.

Conclusions: In light of the findings, maximum, mean elasticity, elasticity ratio of lesions to peripheral parenchyma, and "stiff rim sign" are correlated with the expression of Smad2/3. The combination of the expression of Smad2/3 and "stiff rim sign" might contribute to the diagnosis of breast carcinoma.

Keywords: Breast neoplasms; shear wave elasticity; Smad2/3

Submitted Sep 22, 2021. Accepted for publication Nov 17, 2021.

doi: 10.21037/atm-21-5627

View this article at: https://dx.doi.org/10.21037/atm-21-5627

\section{Introduction}

Breast carcinoma is the primary cause of cancer mortality in females, with an estimated 1 in 8 women developing the disease at some point in their lives (1). Evidence suggests that increased matrix stiffness is a hallmark of breast cancer (2). As an ideal imaging technology based on tissue stiffness, shear wave elasticity (SWE) has been used to quantitatively and qualitatively evaluate the tissue elasticity, showing high sensitivity, specificity, and accuracy in differentiating benign and malignant breast lesions (3).

Transforming growth factor- $\beta$ (TGF- $\beta$ ), a cytokine, participates in antitumor activity, such as proliferation inhibition, but also in carcinogenic processes, such as growth stimulation, increased movement, invasion, and 
metastasis $(4,5)$. Our previous studies have shown that TGF- $\beta$ is associated with maximum elasticity, mean elasticity, the elasticity ratio of lesions to peripheral parenchyma (5). The 2 members of the Smad family, Smad2 and the analogous protein Smad3, mediate the signal transduction of TGF- $\beta$ (6). Further, TGF- $\beta$ binds to transmembrane serine threonine kinase receptors, causing downstream phosphorylation and activation of Smad2 and Smad3, which are then transferred to the nucleus to control the transcription of target genes $(7,8)$. Data from several studies has suggested that the expression of $\operatorname{Smad} 2 / 3$ in malignant tumors is closely related to neoplasm progression, metastasis, and poor prognosis (9). Moreover, several lines of evidence have previously linked Smad2/3 to epithelialmesenchymal transition (EMT) and vasculogenic mimicry (VM) formation in breast cancer $(10,11)$. Both Smad2 and Smad3 are also known to control the expression of a number of dermal extracellular matrix (ECM) components, including collagen and fibronectin $(12,13)$. In previous research, collagen and elastic fiber content were shown to be closely related to the elastic characteristic of breast diseases, which may have a pivotal role in stiffness of breast neoplasm $(14,15)$.

Nevertheless, although a number of cross-sectional studies have suggested that abnormal expression of $\operatorname{Smad} 2 / 3$ can affect the occurrence and development of breast cancer, no study has proved whether $\operatorname{Smad} 2 / 3$ is related to the elastic characteristics of breast lesions. Thus, this study aimed to investigate the relationship between shear wave elasticity and the expression of $\mathrm{Smad} 2 / 3$ in breast lesions. We present the following article in accordance with the STARD reporting checklist (available at https://dx.doi. org/10.21037/atm-21-5627).

\section{Methods}

\section{Patients}

From April 2018 to October 2018, a total of 130 women with 135 consecutive breast lesions were recruited to the study, of which 5 patients had 2 masses. The average age of participants was 44 years (18-73 years). The maximum diameter of the lesion was 0.4 to $5.5 \mathrm{~cm}$ (mean diameter \pm normalized, $1.6 \pm 0.8 \mathrm{~cm}$ ). All lesions were examined by shear wave elastography (SWE), and the pathology was confirmed by surgery.

The inclusion criteria were as follows: (I) pathological findings were available and definite, (II) patients underwent surgery. Patients were excluded if they met the following criteria: (I) had received neoadjuvant chemotherapy or radiotherapy, (II) lacked clinical, pathological, and prognostic follow-up data.

A protocol was prepared before the study without registration. All procedures performed in this study involving human participants were in accordance with the Declaration of Helsinki (as revised in 2013). The prospective study was approved by the Ethics Committee of the Chinese PLA General Hospital (No. S2020-336-01) and informed consent was taken from all the patients.

\section{SWE examinations}

The Aixplorer ${ }^{\circledR}$ ultrasonic diagnostic system (SuperSonic Imagine, Aix-en-Provence, France), with a transducer frequency of 4-12 MHz, was adopted for routine ultrasound (US) and SWE inspections. After the lesion was clearly displayed on the two-dimensional (2D) view, the SWE mode was switched to allow dual dynamic observation of $2 \mathrm{D}$ images and elastograms. The appropriate size and position of the sampling frame was selected to ensure the lesion area and normal gland tissue were at the same level, with a relatively fixed probe position and strength for more than 3 seconds. When recording images, the region of interest (ROI) needed to be selected for calculation of the elasticity value, and the sampling frame covered the entire lesion as much as possible, particularly the hardest part of the lesion. For each patient, the maximum, average, minimum elasticity, elasticity ratio of the lesion to the peripheral parenchyma, and the presence of "stiff rim sign" were measured and recorded 3 times.

\section{Domain-specific antibodies aiming at the phosphorylated Smad2 and Smad3}

Polyclonal anti-phosphorylated Smad2 and antiphosphorylated Smad3 antibodies (abs) were proposed for the phosphorylation junction region and $\mathrm{COOH}$-terminal region of Smad2 and Smad3 by immunization of rabbits with synthetic peptides (16). The relevant antisera were affinity purified with the phosphopeptides (17).

\section{Immunobistochemical studies and image analysis}

After formalin fixation and paraffin embedding, the lesion was cut into $4 \mathrm{~m}$ thick sections. We then detected Smad2/3 in the breast lesions by immunohistochemistry (IHC) as 
Table 1 The elasticity parameters and Smad2/3 in benignity and malignancy

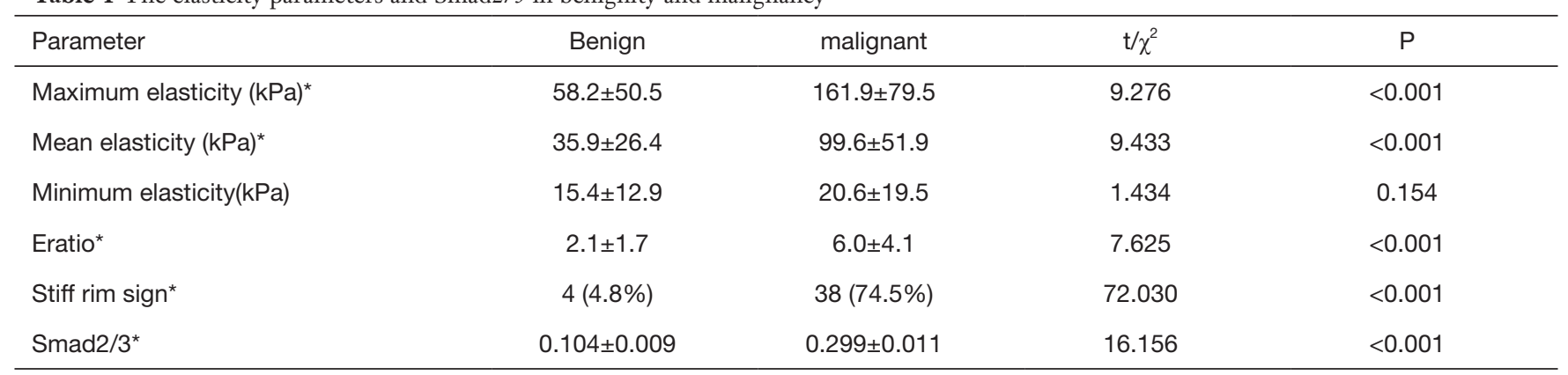

*, a value of $\mathrm{P}<0.05$ is considered statistically significant. Eratio, the elasticity ratio of the lesions to the peripheral tissue.

described previously (9). The software Image-Pro Plus 6.0 (Media Cybernetics, Rockville, MD, USA) was used for image analysis. From each slice, 5 areas of interest were taken under the visual field of 400 . The integrated optical density $(\mathrm{OD})$ and area of the region were measured, and the average OD (integrated optical density/area) was calculated. The average OD was used to evaluate the expression level of $\operatorname{Smad} 2 / 3$.

\section{Statistical methods}

The statistical software SPSS 26.0 (IBM Corp., Armonk, NY, USA) was used for data analysis. Measurement data was described as mean $\pm \mathrm{SD}$ and count data as a percentage (\%). Quantitative data was tested by $\chi^{2}$ test, and qualitative data was tested by independent sample $t$-test. A receiver operating characteristic (ROC) curve was constructed, and the area under curve (AUC) was used to evaluate the elasticity characteristics, expression level of Smad2/3, and their combined differential diagnosis efficiency for mammary malignant and benign diseases. The $Z$ test was adopted to compare the difference of AUCs. Pearson correlation analysis was performed to evaluate the correlation between Smad2/3 expression level and the elastic parameters of breast lesions. A difference of $\mathrm{P}<0.05$ was considered statistically significant.

\section{Results}

\section{Pathology}

The pathological results of 135 breast lesions revealed that 84 were benign (62.2\%) and 51 malignant (37.8\%). The benign lesions comprised 40 fibroadenosis, 32 fibroadenomas, 8 inflammations, 3 papillomas, and 1 benign phyllodes tumors. The malignant lesions involved
46 invasive ductal carcinoma, 3 ductal carcinoma in situ (DCIS), and 2 mucinous carcinomas.

\section{Comparison of elasticity between benignity and malignancy}

Table 1 shows the maximum, mean, minimum elasticity, elasticity ratio of lesions to peripheral parenchyma of lesions, and detection rate of "stiff rim sign" of benignity and malignancy. Malignant nodules presented significantly higher maximum, mean elasticity, elasticity ratio of lesions to peripheral parenchyma, and detection rate of "stiff rim sign" than benign nodules $(\mathrm{P}<0.001$, Figure $1 A, 1 B)$.

\section{Comparison of Smad2/3 expression level between benignity and malignancy}

In all the breast lesions, Smad2/3 had an average OD of $0.142 \pm 0.105$. Among them, the expression level of smad2/3 in malignant breast tissues was significantly higher than that in benign breast tissues $(0.299 \pm 0.011 \mathrm{vs}$. $0.104 \pm 0.009, \mathrm{P}<0.001$, Table 1, Figure $2 A, 2 B)$. The AUC of the differential diagnosis of benignity and malignancy by Smad2/3 expression level was 0.957 (0.906-0.985, Figure 3). When the mean OD cutoff value was 0.1495 , the sensitivity and specificity of differential diagnosis of benignity and malignancy were $94.00 \%$ and $91.36 \%$, respectively.

\section{Correlation between elasticity and Smad2/3 expression level}

The study showed that the expression level of smad $2 / 3$ was positively correlated with the maximum elasticity $(\mathrm{r}=0.657$, Figure $4 A$ ), the average elasticity $(\mathrm{r}=0.640$, Figure $4 B)$, and the ratio of lesions to peripheral tissue ( $\mathrm{r}=0.470$, Figure $4 C)$. The expression level of Smad2/3 in breast lesions with "stiff rim sign" was significantly higher than that of those without 

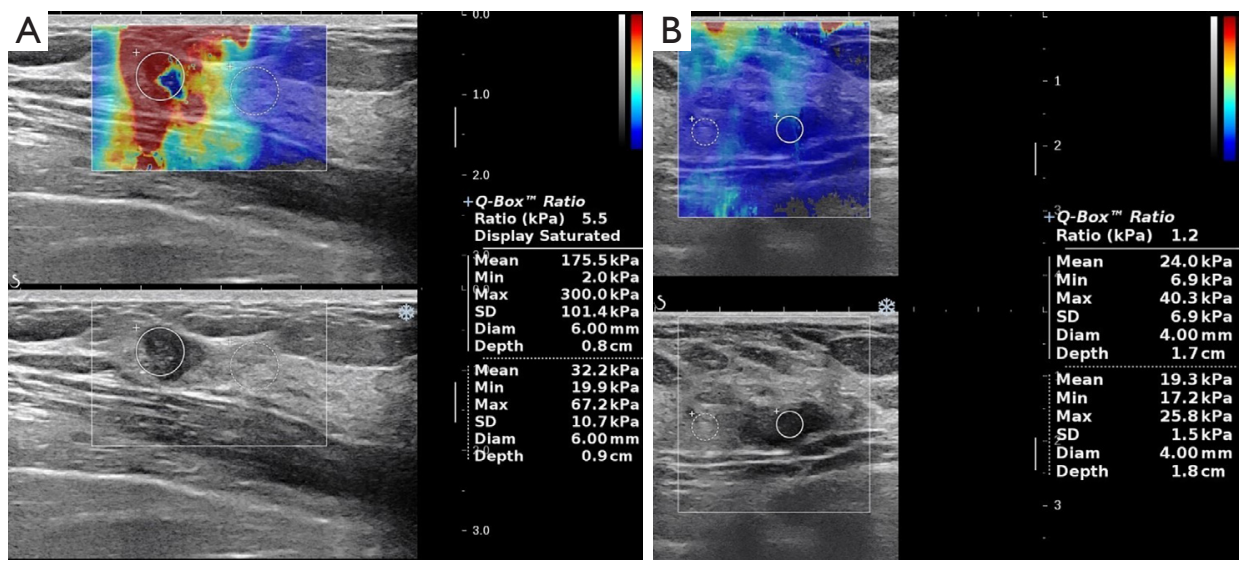

Figure 1 Elastic parameters of breast lesion. (A) SWE image of an invasive ductal carcinoma in a 43-year-old female. The maximum elasticity was $300.0 \mathrm{kPa}$, the average elasticity was $175.5 \mathrm{kPa}$, and the elasticity ratio of lesions to peripheral parenchyma was 5.5 ; (B) SWE image of a fibroadenoma in a 56-year-old female. The maximum elasticity was $49.3 \mathrm{kPa}$, the average elasticity was $24.0 \mathrm{kPa}$, and the elasticity ratio of lesions to peripheral parenchyma was 1.2. SWE, shear wave elastography; $\mathrm{kPa}$, kilopascal.
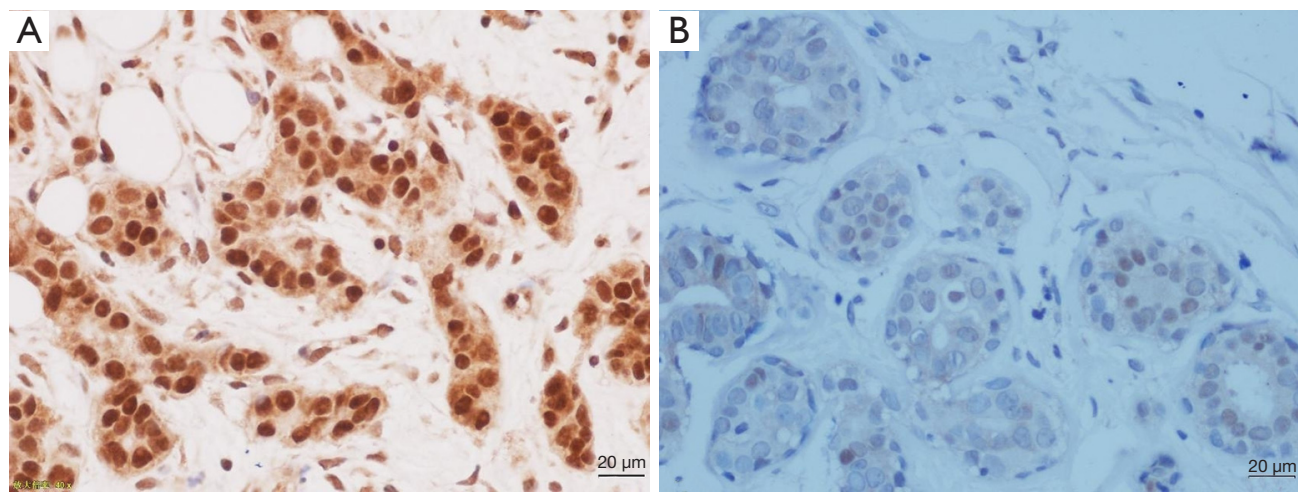

Figure 2 The staining method was immunohistochemical staining with an amplification of 400 times. (A) Smad2/3 expression was strongly positive with an average optical density of 0.2824 ; (B) Smad2/3 was weakly expressed mainly in cytoplasm and nucleus, with an average optical density of 0.0220 .

“stiff rim sign" (0.2512 \pm 0.076 vs. $0.075 \pm 0.050)$.

\section{Diagnostic properties of Smad2/3 combined with elasticity}

Table 2 lists the diagnostic properties of Smad2/3, the maximum, mean elasticity, elasticity ratio of lesions to peripheral parenchyma of lesions, and detection rate of "stiff rim sign" and Smad2/3 combined with "stiff rim sign". Although the Smad2/3 combined with "stiff rim sign" achieved no higher specificity than "stiff rim sign" $(\mathrm{P}<0.05)$, it yielded the significantly highest diagnostic sensitivity and accuracy in all indexes $(\mathrm{P}<0.05$ for all) (Table 2).

\section{Discussion}

Accumulating evidence has demonstrated that SWE has excellent diagnostic capabilities in distinguishing benign and malignant lump masses, which is conducive to the earlier diagnosis of mammary cancer $(18,19)$. Collagen and fibronectin in ECM, as important factors determining lesion elasticity, play crucial roles in the occurrence and development of breast cancer $(14,15)$. However, the Smad2/3 pathway, as a major pathway controlling the expression of collagen and fibronectin (13), has rarely been studied in relation to the elastic characteristics of breast lesions.

This study indicated that malignant neoplasms exhibit 
significantly higher maximum, mean elasticity, and elasticity ratio of lesions to peripheral parenchyma than benign neoplasms, consistent with previous research $(19,20)$. Breast tumors have been reported to develop with ECM stiffening,

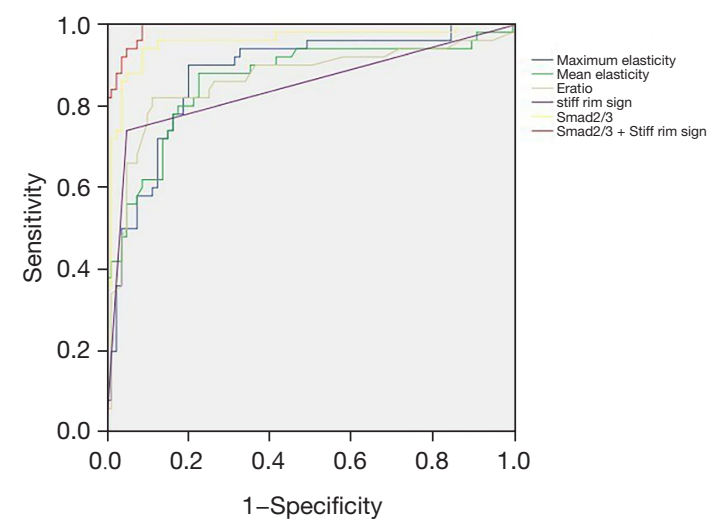

Figure 3 The ROC curve of the differentiating diagnosis of benignity and malignancy by $\operatorname{Smad} 2 / 3$ expression level, the elastic characteristics of lesions, "stiff rim sign" and Smad2/3 combined with "stiff rim sign". Eratio, the elasticity ratio of the lesions to the peripheral tissue.
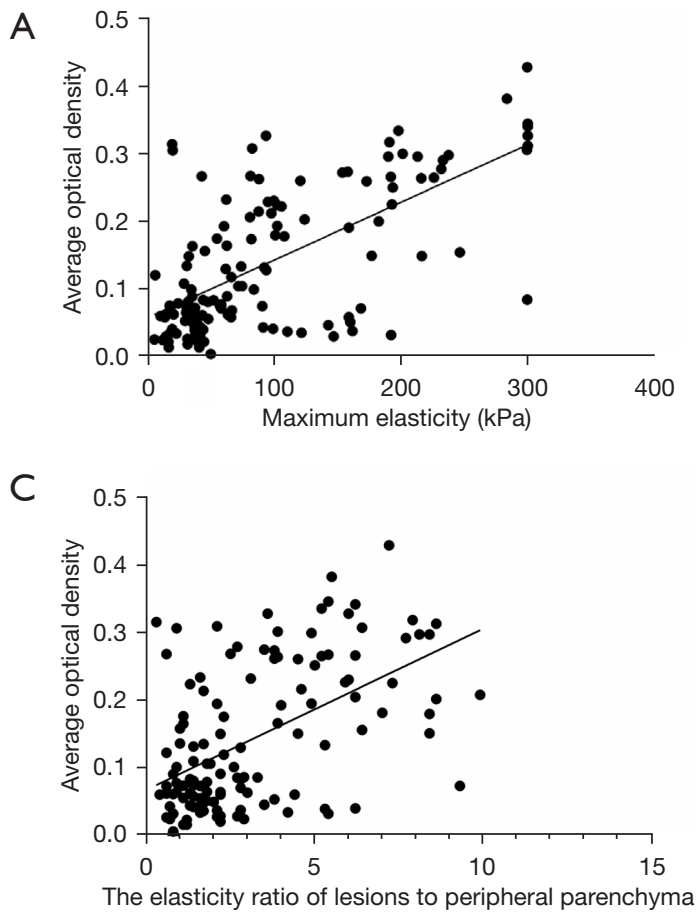

collagen cross-linking, increased focal adhesions, fibrogenic response, and tumor infiltration into adjacent stroma, which were shown to be responsible for the scirrhous properties of tumors (21-23). Our previous study showed that the diagnostic sensitivity and specificity of US + SWE for breast lesions could reach $88.6 \%$ and $90.5 \%$, respectively (20). Also, this research showed that maximum, mean elasticity, elasticity ratio of lesions to peripheral parenchyma, and detection rate of "stiff rim sign" all had high diagnostic efficiency, which further confirmed that SWE was of great value in the differential diagnosis of breast neoplasms.

This study revealed that the expression of $\mathrm{Smad} 2 / 3$ in benign breast nodules was significantly less than that in malignant masses, which was defined as a critical feature for the recognition of malignant masses. Previous studies have gradually revealed that the $S \operatorname{mad} 2 / 3$ pathway could mediate anti-tumor and carcinogenic effects of TGF- $\beta$, and that the Smad $2 / 3$ pathway mediates the anti-tumor and carcinogenic effects of TGF- $\beta$ depending on the cell's progression stage and other synergistic contextual changes $(6,10)$. Studies have reported that $S \operatorname{mad} 2 / 3$ is overexpressed in breast cancer (24), pancreatic cancer (25), gastric cancer (26) and other tumors $(27,28)$, which can be used to indicate
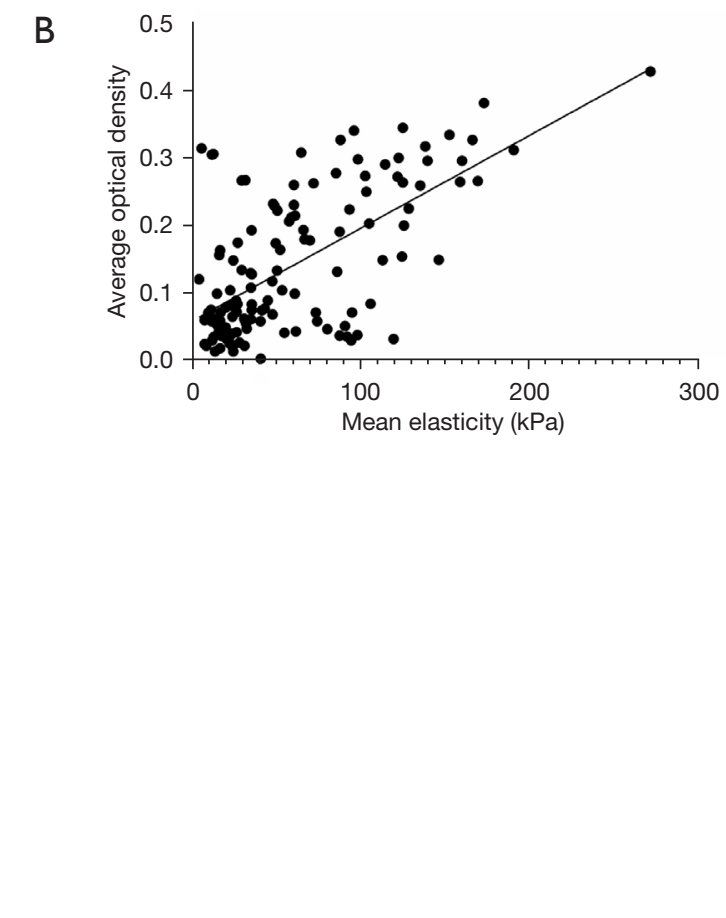

Figure 4 Correlation between Smad2/3 expression level and elastic characteristics of lesions. (A) The correlation between Smad2/3 expression level and maximum elasticity was shown; (B) the correlation between Smad2/3 expression level and mean elasticity was shown; (C) the correlation between Smad2/3 and the ratio of elasticity between lesions and peripheral tissues was shown. 
Table 2 Sensitivity, specificity, Youden index and accuracy of the parameters of SWE, the expression level of Smad2/3 and the combination of Smad2/3 and "stiff rim sign"

\begin{tabular}{|c|c|c|c|c|c|}
\hline Parameter & Cutoff value (kPa) & Sensitivity (\%) & Specificity (\%) & Youden index & Accuracy (\%) \\
\hline Mean elasticity & $>47.9$ & 88.24 & 77.38 & 0.6562 & 81.48 \\
\hline Eratio & $>3$ & 82.35 & 87.95 & 0.7030 & 85.83 \\
\hline Stiff rim sign & - & 74.51 & 95.24 & 0.6975 & 87.41 \\
\hline Smad2/3 combined with stiff rim sign & $>0.194$ & 100.00 & 91.25 & 0.9125 & 94.56 \\
\hline
\end{tabular}

Eratio, the elasticity ratio of the lesions to the peripheral tissue; SWE, shear wave elastography.

the prognostic effect of those malignant tumors. A study by Yamagata et al. (4) highlighted a significant increase in c-Jun NH2-terminal kinase (JNK)-dependent Smad2/3 as the process of neoplasia progressed from normal colorectal epithelial cells to invasive adenocarcinoma with distant metastasis. Additionally, knocking out the expression of Smad2/3 inhibited the expression of Snail, vimentin, fibronectin, and cancer cell invasion induced by epidermal growth factor (EGF), indicating that the activation of Smad2/3 was associated with EGF-induced EMT in breast cancer (10). Increased expression of $\operatorname{Smad} 2 / 3$ maintains the stem cell characteristics of breast cancer cells, which in turn promotes the proliferation and progression of breast cancer (24). Furthermore, vasculogenic mimicry (VM) formation, via the $\operatorname{Smad} 2 / 3$ pathway, could support growing tumors and promote disease progression in vitro and in vivo (11). It has been shown that by activating the Smad2/3 pathway, the tumorigenicity and sphere-forming capability of breast cancer cells could be enhanced (29). Thus, in this study, the findings of the higher expression of Smad2/3 in malignant breast lesions than that of benign lesions indicated that $S m a d 2 / 3$ may be partially responsible for the development of breast carcinoma.

This study revealed that the expression of $S \operatorname{mad} 2 / 3$ was related to the maximum, mean elasticity, and elasticity ratio of lesions to peripheral parenchyma of lesions. Literature has demonstrated that the expression of Smad2 and Smad3 is upregulated in cirrhotic livers compared with normal livers (30). In human atherosclerotic lesions, genes activated by TGF- $\beta /$ Smad signaling that have the potential to affect lesion characteristics include the $\alpha 1$ chain of type I collagen, $\alpha 2$ chain of type I collagen, $\alpha 1$ chain of type III collagen, and connective tissue growth factor (13). A study demonstrated that Smad2 and Smad3 knockdown alleviated the synthesis of collagen I, V, IV, and fibronectin messenger RNA (mRNA) in fibroblast cells both in vitro and in adult mice (31). Moreover, it has been shown that $\operatorname{Smad} 2 / 3$ also contributes to inhibiting the degradation of proteins (collagen and fibronectin) in EGM $(21,32)$. Furthermore, via the Smad2/3 pathway, various ECM-remodeling enzymes are induced in breast cancer, which could improve cell adhesion and reduce the activity of the lesion (33). Therefore, this correlation between Smad2/3 and elastic characteristics of breast lesions suggests that $\operatorname{Smad} 2 / 3$ might have a detrimental effect on the formation of tumor stiffness.

In this study, the incidence of "stiff rim sign" in malignant tissues $(74.5 \%)$ was significantly greater than that in benign tissues $(4.8 \%)(\mathrm{P}<0.001)$, and compared with breast lesions without "stiff rim sign", those with "stiff rim sign" showed higher Smad2/3 expression level, suggesting the expression level of $S \mathrm{mad} 2 / 3$ was also associated with "stiff rim sign". Research has shown that the "stiff rim sign" might stem from the proliferation of connective tissue or the involvement by tumor cells into the peripheral organization, which is more common in malignant breast lesions (20). Also, a previous study showed that fibroblasts and other progenitor cells were converted into myofibroblasts via the Smad2/3 pathway (34). In the highly vascularized desmoplasia of breast cancers, myofibroblast activity shifts outward to the growing edge, producing a 2-fold to more than 10-fold increase in the stiffness of tissues surrounding the tumor (23). Thus, "stiff rim sign" was associated with $S \mathrm{mad} 2 / 3$ expression level, suggesting that $\mathrm{Smad} 2 / 3$ might participate in the formation process of "stiff rim sign".

Previous studies have shown that the "stiff rim sign" held a high diagnostic competence $(20,35)$. In this study, the 
specificity of Smad2/3 combined with "stiff rim sign" was lower than that of "stiff rim sign"; however, its diagnostic sensitivity and accuracy was significantly greater than that of "stiff rim sign" ( $\mathrm{P}<0.05$ for all). The combination between Smad2/3 and "stiff rim sign" might be a new entry point for the differential diagnosis of breast cancer, which may provide a supplement to the diagnosis of breast lesions.

This study has several limitations. First, the differential role of Smad2 and Smad3 in the process of increasing stiffness of tumor tissue has not been studied. The phenotype of Smad2 and Smad3 knockout mice and the comparison of the activation of Smad2 and Smad3 of TGF- $\beta$ downstream genes indicated that Smad 2 and Smad 3 had different functions in TGF- $\beta$ signal transduction and may play varied roles in the formation of tumor stiffness (6). Second, which regions (N-terminal Mad homology domain-1, intermediate linker, C-terminal Mad homology domain-2) of Smad2/3 had been phosphorylated was not detected. It has been reported that late-stage cancer with deep invasion and/or metastasis is typically characterized by high phosphorylation of Smad3 linker region in colorectal cancer (4), and the relationship between high phosphorylation of distinct roles and the stiffness of breast lesions needs further investigation.

\section{Conclusions}

In conclusion, maximum, mean elasticity, elasticity ratio of lesions to peripheral parenchyma, and "stiff rim sign" are correlated with the expression of Smad2/3, suggesting $\operatorname{smad} 2 / 3$ may play a vital role in leading to tumor stiffness. The combination of the expression of Smad2/3 and "stiff rim sign" might contribute to the diagnosis of breast cancer.

\section{Acknowledgments}

Funding: This work was supported by the National Natural Science Foundation (81771832) and Top Project of military Medical Science and Technology Youth Training Program (19QNP071).

\section{Footnote}

Reporting Checklist: The authors have completed the STARD reporting checklist. Available at https://dx.doi. org/10.21037/atm-21-5627

Data Sharing Statement: Available at https://dx.doi. org/10.21037/atm-21-5627

Conflicts of Interest: All authors have completed the ICMJE uniform disclosure form (available at https://dx.doi. org/10.21037/atm-21-5627). All authors report that this work was supported by the National Natural Science Foundation (81771832) and Top Project of military Medical Science and Technology Youth Training Program (19QNP071). The authors have no other conflicts of interest to declare.

Ethical Statement: The authors are accountable for all aspects of the work in ensuring that questions related to the accuracy or integrity of any part of the work are appropriately investigated and resolved. All procedures performed in this study involving human participants were in accordance with the Declaration of Helsinki (as revised in 2013). The prospective study was approved by the Ethics Committee of the Chinese PLA General Hospital (No. S2020-336-01) and informed consent was taken from all the patients.

Open Access Statement: This is an Open Access article distributed in accordance with the Creative Commons Attribution-NonCommercial-NoDerivs 4.0 International License (CC BY-NC-ND 4.0), which permits the noncommercial replication and distribution of the article with the strict proviso that no changes or edits are made and the original work is properly cited (including links to both the formal publication through the relevant DOI and the license). See: https://creativecommons.org/licenses/by-nc-nd/4.0/.

\section{References}

1. Bray F, Ferlay J, Soerjomataram I, et al. Global cancer statistics 2018: GLOBOCAN estimates of incidence and mortality worldwide for 36 cancers in 185 countries. CA Cancer J Clin 2018;68:394-424.

2. Clavel C, Birembaut P, Adnet JJ, et al. Breast carcinomas and the extracellular matrix. Ann Pathol 1988;8:107-13.

3. Xue Y, Yao S, Li X, et al. Benign and malignant breast lesions identification through the values derived from shear wave elastography: evidence for the meta-analysis. Oncotarget 2017;8:89173-81.

4. Yamagata H, Matsuzaki K, Mori S, et al. Acceleration of Smad2 and Smad3 phosphorylation via c-Jun NH(2)terminal kinase during human colorectal carcinogenesis. Cancer Res 2005;65:157-65.

5. Zhang M K, Shang Q J, Li S Y et al. TGF- $\beta 1$ : is it related to the stiffness of breast lesions and can it predict axillary 


\section{Page 8 of 9}

lymph node metastasis?.Ann Transl Med, 2021, 9: 870.

6. Brown KA, Pietenpol JA, Moses HL. A tale of two proteins: differential roles and regulation of Smad2 and Smad3 in TGF-beta signaling. J Cell Biochem 2007;101:9-33.

7. Heldin CH, Moustakas A. A new twist in Smad signaling. Dev Cell 2006;10:685-6.

8. Tian F, DaCosta Byfield S, Parks WT, et al. Reduction in Smad2/3 signaling enhances tumorigenesis but suppresses metastasis of breast cancer cell lines. Cancer Res 2003;63:8284-92.

9. Xie W, Mertens JC, Reiss DJ, et al. Alterations of Smad signaling in human breast carcinoma are associated with poor outcome: a tissue microarray study. Cancer Res 2002;62:497-505.

10. Kim J, Kong J, Chang H, et al. EGF induces epithelialmesenchymal transition through phospho-Smad2/3Snail signaling pathway in breast cancer cells. Oncotarget 2016;7:85021-32.

11. Gong W, Sun B, Zhao X, et al. Nodal signaling promotes vasculogenic mimicry formation in breast cancer via the Smad2/3 pathway. Oncotarget 2016;7:70152-67.

12. Li X, Guo L, Yang X, Wang J, et al. TGF- $\beta 1$-induced connexin 43 promotes scar formation via the Erk/MMP-1/ collagen III pathway. J Oral Rehabil 2020;47 Suppl 1:99-106.

13. Kalinina N, Agrotis A, Antropova Y, et al. Smad expression in human atherosclerotic lesions: evidence for impaired TGF-beta/Smad signaling in smooth muscle cells of fibrofatty lesions. Arterioscler Thromb Vasc Biol 2004;24:1391-6.

14. Liu G, Zhang MK, He Y, et al. Shear wave elasticity of breast lesions: would it be correlated with the extracellular matrix components? Gland Surg 2019;8:399-406.

15. Wang ZL, Sun L, Li Y, et al. Relationship between elasticity and collagen fiber content in breast disease: a preliminary report. Ultrasonics 2015;57:44-9.

16. Matsuzaki K, Kitano C, Murata M, et al. Smad2 and Smad3 phosphorylated at both linker and $\mathrm{COOH}$-terminal regions transmit malignant TGF-beta signal in later stages of human colorectal cancer. Cancer Res 2009;69:5321-30.

17. Furukawa F, Matsuzaki K, Mori S, et al. p38 MAPK mediates fibrogenic signal through Smad3 phosphorylation in rat myofibroblasts. Hepatology 2003;38:879-89.

18. Huang R, Jiang L, Xu Y, et al. Comparative Diagnostic Accuracy of Contrast-Enhanced Ultrasound and Shear Wave Elastography in Differentiating Benign and Malignant Lesions: A Network Meta-Analysis. Front Oncol 2019;9:102.

19. Suvannarerg V, Chitchumnong P, Apiwat W, et al. Diagnostic performance of qualitative and quantitative shear wave elastography in differentiating malignant from benign breast masses, and association with the histological prognostic factors. Quant Imaging Med Surg 2019;9:386-98.

20. Liu G, Zhang MK, He Y, et al. BI-RADS 4 breast lesions: could multi-mode ultrasound be helpful for their diagnosis? Gland Surg 2019;8:258-70.

21. Jena MK, Janjanam J. Role of extracellular matrix in breast cancer development: a brief update. F1000Res 2018;7:274.

22. Kaushik N, Kim S, Suh Y, et al. Proinvasive extracellular matrix remodeling for tumor progression. Arch Pharm Res 2019;42:40-7.

23. Insana MF, Pellot-Barakat C, Sridhar M, et al. Viscoelastic imaging of breast tumor microenvironment with ultrasound. J Mammary Gland Biol Neoplasia 2004;9:393-404.

24. Yang R, Liang J, Xu GX, et al. Human cytomegalovirus glycoprotein $\mathrm{B}$ inhibits migration of breast cancer MDAMB-231 cells and impairs TGF- $\beta / \mathrm{Smad} 2 / 3$ expression. Oncol Lett 2018;15:7730-8.

25. Kang JH, Jung MY, Leof EB. B7-1 drives TGF- $\beta$ stimulated pancreatic carcinoma cell migration and expression of EMT target genes. PLoS One 2019;14:e0222083.

26. Pang T, Yin X, Luo T, et al. Cancer-associated fibroblasts promote malignancy of gastric cancer cells via Nodal signalling. Cell Biochem Funct 2020;38:4-11.

27. Matveeva A, Kovalevska L, Kholodnyuk I, et al. The TGFbeta - SMAD pathway is inactivated in cronic lymphocytic leukemia cells. Exp Oncol 2017;39:286-90.

28. Zhao M, Mishra L, Deng CX. The role of TGF- $\beta$ / SMAD4 signaling in cancer. Int J Biol Sci 2018;14:111-23.

29. Gong W, Sun B, Sun H, et al. Nodal signaling activates the $S$ mad2/3 pathway to regulate stem cell-like properties in breast cancer cells. Am J Cancer Res 2017;7:503-17.

30. Honjo M, Moriyasu F, Sugimoto K, et al. Relationship between the liver tissue shear modulus and histopathologic findings analyzed by intraoperative shear wave elastography and digital microscopically assisted morphometry in patients with hepatocellular carcinoma. J Ultrasound Med 2014;33:61-71.

31. Huang S, Chen B, Humeres C, et al. The role of Smad2 and $\mathrm{Smad} 3$ in regulating homeostatic functions of fibroblasts in vitro and in adult mice. Biochim Biophys Acta Mol Cell Res 2020;1867:118703.

32. Kawecki M, Łabuś W, Klama-Baryla A, et al. A review of decellurization methods caused by an urgent need for quality control of cell-free extracellular matrix' scaffolds and their role in regenerative medicine. J Biomed Mater 
Res B Appl Biomater 2018;106:909-23.

33. Kim HJ, Park JH, Shin JM, et al. TGF- $\beta 1$-induced HSP47 regulates extracellular matrix accumulation via Smad2/3 signaling pathways in nasal fibroblasts. Sci Rep 2019;9:15563. Erratum in: Sci Rep 2020;10:9585.

34. Barr RG. Shear wave imaging of the breast: still on the

Cite this article as: Zhang MK, Li SY, Xue SW, Liu G, Wang ZL. Correlation of elastic characteristics of breast lesions with the expression of Smad2/3. Ann Transl Med 2021;9(22):1681. doi: 10.21037/atm-21-5627 learning curve. J Ultrasound Med 2012;31:347-50.

35. Wang ZL, Li Y, Wan WB, et al. Shear-Wave Elastography: Could it be Helpful for the Diagnosis of Non-Mass-Like Breast Lesions? Ultrasound Med Biol 2017;43:83-90.

(English Language Editor: J. Jones) 